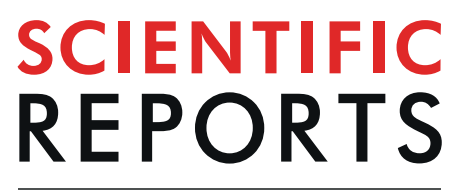

natureresearch

There are amendments to this paper

\title{
Detection of Neorickettsia risticii, the agent of Potomac horse fever, in horses from Rio de Janeiro, Brazil
}

\author{
Patrícia Gonzaga Paulino ${ }^{1 凶}$, Nádia Almosny², Renata Oliveira², Vanessa Viscardi², \\ Ananda Müller ${ }^{3,4}$, Andresa Guimarães $\mathbb{D}^{5}$, Cristiane Baldani ${ }^{5}$, Claudia da Silva ${ }^{6}$, \\ Maristela Peckle ${ }^{6}$, Carlos Massard ${ }^{6}$ \& Huarrisson Santos ${ }^{1}$
}

This study aims to report the presence of Neorickettsia risticii DNA in blood samples from naturally infected horses in Rio de Janeiro, provide clinicopathological findings related to the infection, and report the phylogenetic diversity of the $16 \mathrm{~S}$ rDNA of $N$. risticii in order to evaluate its heterogeneity. Real-time quantitative polymerase chain reaction (qPCR) was performed to investigate the presence of $N$. risticii in samples collected from horses $(n=187)$. Five positive samples were found in the molecular screening. Hypoalbuminemia and high levels of creatine kinase and lactate dehydrogenase were the predominant findings in the biochemical analysis. The sequences were similar to those of $N$. risticii. Phylogenetic analysis revealed genotype segregation based on the geographical distribution in the $N$. risticii sequence clade. Dendrograms constructed with five hypervariable regions revealed that V4 distinguished Neorickettsia at the species level and produced a phylogeny that best represented the phylogeny obtained with the complete $16 \mathrm{~S}$ rDNA sequence. This is the first report of $N$. risticii DNA in the blood of Brazilian horses based on sequences deposited in GenBank. Further studies are necessary to clarify the epidemiological chain of this vector-borne parasite in order to determine and establish appropriate preventive measures in the equine trading market.

Potomac horse fever (PHF) is an acute systemic and potentially fatal disease of horses, which is also known as equine monocytic ehrlichiosis. It is caused by Neorickettsia risticii, an obligate intracellular endosymbiotic bacterium of digeneans (Platyhelminthes, Digenea) that parasitize snails and insects ${ }^{1,2}$. This disease is normally acquired through the accidental ingestion of insects or snails containing encysted trematodes infected with $N$. ris$\mathrm{ticii}^{3}$. Once the bacterium is released into the lumen of a horse's gastrointestinal tract, it invades and multiplies in colon epithelial cells. It translocates into the blood and infects monocytes, mast cells, and macrophages ${ }^{4}$. Clinical presentation can vary from moderate to severe with non-specific clinical signs, which can include fever, lethargy, depression, laminitis, diarrhoea, and anorexia followed by severe dehydration ${ }^{5}$. Haematological and biochemical alterations, such as electrolyte loss, haemoconcentration, and prerenal azotemia, may also be observed in horses infected by N. risticii ${ }^{6}$.

Case descriptions from southern Brazil show that PHF may be transmitted by blood transfusion and by the ingestion of snails from the genus Heleobia ${ }^{7}$. Reports from Brazil, Uruguay, and the United States have suggested that the disease has a seasonal presentation (usually occurring in the summer and beginning of fall), although it may occur in any season depending on weather conditions ${ }^{7,8}$.

The disease has been geographically well reported in Canada $^{9}$ and the United States ${ }^{1,6}$. Additionally, the DNA of N. risticii has been detected in bats from Argentina in South America ${ }^{10,11}$. In Brazil, the pathogen has been detected only at the molecular level in horses from the South Region ${ }^{2-13}$. Although the circulation of this pathogen has been confirmed in the South Region of Brazil, no N. risticii sequences are available in GenBank, and the presence of $N$. risticii DNA in other Brazilian regions has not been confirmed. Some serological studies have

${ }^{1}$ Department of Epidemiology and Public Health, Federal Rural University of Rio de Janeiro (UFRRJ), BR 465, Km 7, Seropedica, RJ, 23890000, Brazil. 'Department of Veterinary Clinic and Pathology, Federal Fluminense University, Niteroi, Brazil. ${ }^{3}$ Ross University School of Veterinary Medicine, Basseterre, Saint Kitts and Nevis. ${ }^{4}$ Instituto de Ciencias Clínicas Veterinarias, Universidad Austral de Chile, Valdivia, Chile. ${ }^{5}$ Department of Veterinary Medicine and Surgery, Veterinary Institute, Federal Rural University of Rio de Janeiro (UFRRJ), BR 465, Km 7, Seropedica, RJ,

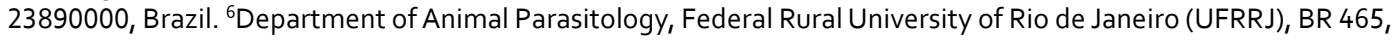
Km 7, Seropedica, RJ, 23890000, Brazil. ${ }^{凶}$ e-mail: patgpaulino@gmail.com 


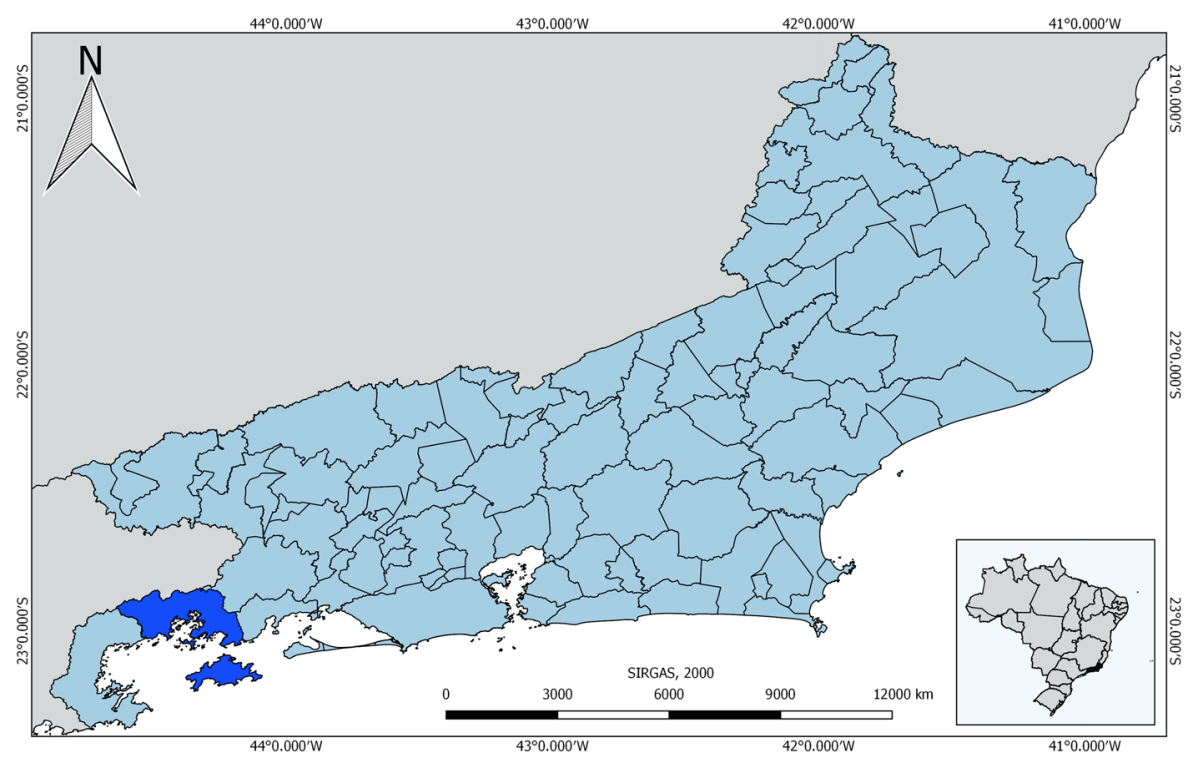

Figure 1. The state of Rio de Janeiro, Brazil and the location of the Angra dos Reis municipality, where blood samples were collected from horses. The colour Blue indicates the municipality of Angra dos Reis and in Grey are the bordering states. Scale bar $=20 \mathrm{Km}$.

suggested that $N$. risticii circulates in horses in the states of Sao Paulo and Rio de Janeiro (Southeast Region) $)^{14-16}$. However, due to the possibility of cross-reaction with other organisms from the Anaplasmataceae family, the confirmation of findings using DNA detection-based methods is required.

The $16 \mathrm{~S}$ rDNA is key to the taxonomic organization of the Anaplasmataceae family. The Neorickettsia group is the most divergent genetic cluster of the Ehrlichieae ${ }^{17}$. For example, in N. risticii sequences, $16 \mathrm{~S}$ rDNA may be divergent by as many as 15 nucleotides, highlighting the phylogenetic heterogeneity of this group ${ }^{17}$. The $16 \mathrm{~S}$ rDNA is highly conserved and usually has more than $97 \%$ sequence identity ${ }^{18}$. This $3 \%$ divergence is concentrated mainly in nine hypervariable regions that are taxon-specific and need to be determined for novel organisms by sequence analysis of the complete molecule ${ }^{18}$. However, only a few studies have employed complete $16 \mathrm{~S}$ rDNA sequences due to the quality restraint of sequencing technology. Hence, the most suitable hypervariable region of $16 \mathrm{~S}$ rDNA is important for phylogenetic studies of bacteria.

In areas where $N$. risticii circulates, it has a great economic impact on equine production. Local veterinarians need to know that PHF occurs so that they may consider it as a differential diagnosis for fever, depression, diarrhoea, and anorexia ${ }^{19}$. Therefore, it is very important to investigate, report, and confirm cases in areas where the disease status is unknown in order to apply preventive measures. Hence, the present study aims to detect $N$. risticii at the molecular level in whole blood samples of naturally infected horses from the state of Rio de Janeiro, Brazil, provide clinicopathological findings related to the infection, and report the phylogenetic diversity of the $16 \mathrm{~S}$ rDNA of $N$. risticii in order to evaluate its heterogeneity.

\section{Results}

The frequency of $N$. risticii in horses from Angra dos Reis in the state of Rio de Janeiro (Fig. 1) in the Southeast Region of Brazil was $2.67 \%$ (5/188). All positive samples were successfully sequenced and presented $99.53 \%$ to $100 \%$ similarity to other $N$. risticii sequences from GenBank and the SILVA database. The percent identity between the sequences obtained in the present study ranged from 99.92 to $100 \%$.

Regarding clinicopathological findings, infected animals did not show abnormalities in the erythrogram, except that one presented with anaemia and thrombocytopenia according to the reference values (Supplementary Table S1). However, 60\% (3/5) of infected horses showed high levels of fibrinogen. Among these horses, one presented with leukocytosis, eosinophilia, and basophilia. Another presented with lymphocytosis, and the final animal presented with only eosinophilia (Supplementary Table S1). Biochemical analysis showed that all infected horses presented with hypoalbuminemia and high Lactate Dehydrogenase and Creatine Kinase activity, while two showed hypoproteinemia (Supplementary Table S1). Only one horse had hyperglobulinemia. Urea, creatinine, and aspartate aminotransferase (AST) were within the reference range in all positive animals.

The phylogenetic analysis of $N$. risticii based on $16 \mathrm{~S}$ rDNA clearly showed that the five sequences found in this study were grouped with other $N$. risticii sequences deposited in GenBank. Based on an evolutionary distance of $0.3 \%$, the N. risticii group showed three geographically distinguishable genotypes. These were Genotype A (six sequences), Genotype B (four sequences), and Genotype C (seven sequences; Fig. 2). Genotype A has composed of $N$. risticii sequences from Brazil and Argentina; Genotype B was composed of $N$. risticii sequences from Illinois, Ohio, and Pennsylvania in the United States; and Genotype C was composed of N. risticii from California in the United States. The N. risticii genotype identified in South America (that is, the one identified in Brazil and Argentina) was more closely related to the California genotype $(0.3 \%$ distance $)$ than to the Illinois, Ohio, and Pennsylvania genotype ( $0.5 \%$ distance) (Supplementary Fig. S1). 


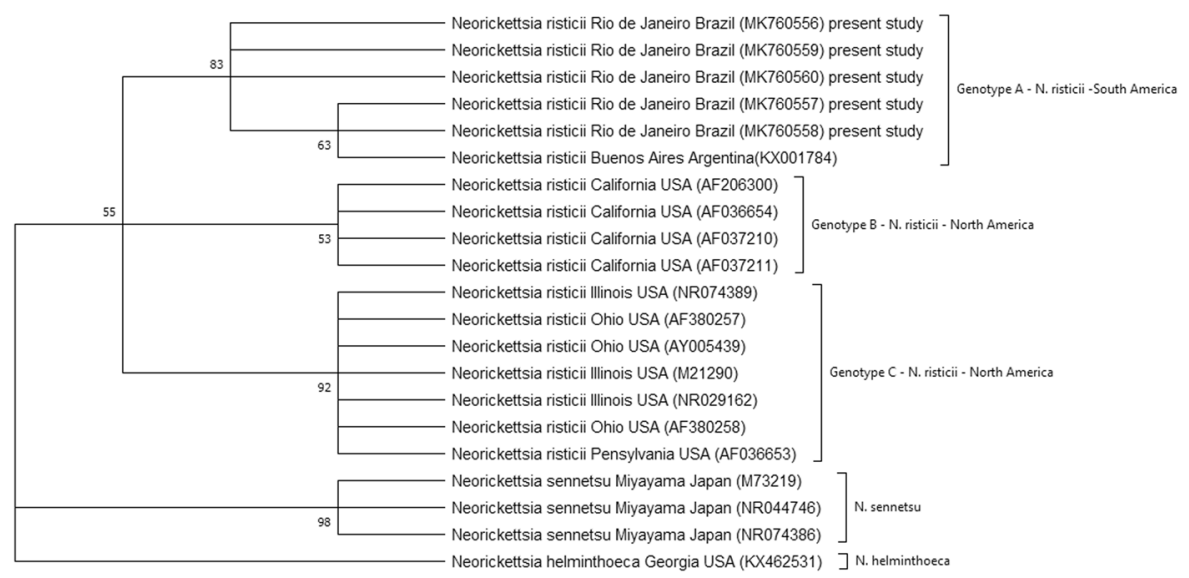

Figure 2. Phylogenetic analysis of the Neorickettsia risticii based on $16 \mathrm{~S}$ rDNA sequence comparison. GenBank accession numbers have been shown in parentheses. The tree was constructed using the maximum likelihood method, and numbers above internal nodes indicate the percentages of 1000 bootstrap replicates that supported the branch.

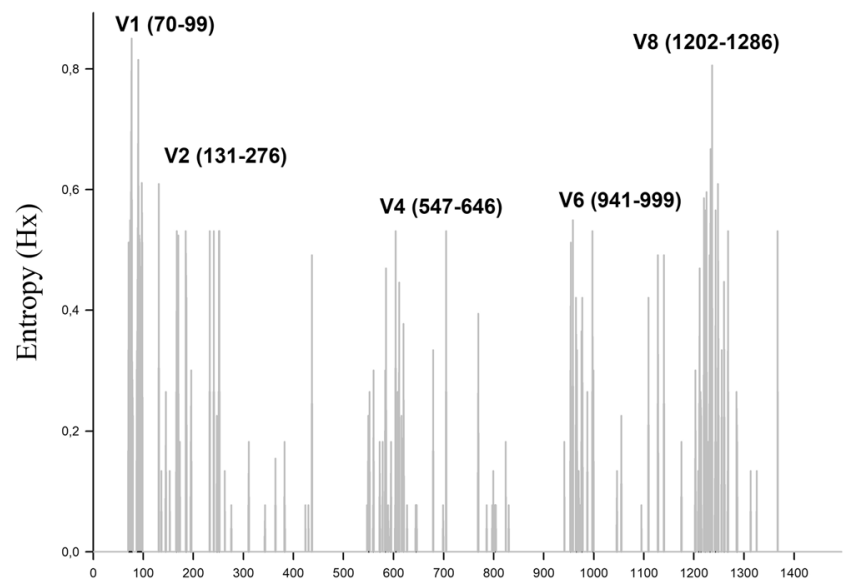

Alignment Position (Residue Number)

Figure 3. Entropy plot of $16 \mathrm{~S}$ rRNA gene sequence alignment from Neorickettsia risticii. Hypervariable regions have been indicated per Chakravorty et al. (2007). The sequence area presented excluded poorly supported areas from the beginning and end of the sequences and thus excluded the V9 region.

Entropy analysis revealed five hypervariable regions (V1, V2, V4, V6, and V8) in the 16S rDNA sequence alignment of Neorickettsia spp. (Fig. 3). Dendrograms constructed with the five hypervariable regions revealed that V4 was the region that distinguished Neorickettsia at the species level and produced the phylogeny that best represented that obtained from the complete 16S rDNA sequence (Supplementary Figs. S2-S6). Hypervariable regions V1, V2, V6, and V8 were less suitable for species identification due to a higher degree of sequence conservation. No region could distinguish the $N$. risticii genotypes. It was impossible to determine the variable region V9 of Neorickettsia due to a lack of nucleotide sequences in the $5^{\prime}$ terminal portions.

\section{Discussion}

The present study is the first to report the presence of $N$. risticii DNA in naturally infected horses in the Southeast Region of Brazil, in the state of Rio de Janeiro. Previous studies performed in the South Region of Brazil reported the presence of $N$. risticii in horses ${ }^{8-13}$. However, these reports were based on serology or PCR methods without further confirmation from either sequencing or culturing of the bacterium ${ }^{2}$. Although serological studies have been conducted in several localities in the Southeast Region of Brazil, to date, there have been no molecular reports confirming the presence of N. risticii DNA in animal samples. According to the literature, $22.2 \%$ of seroreactive horses were found in the mountains and metropolitan areas of Rio de Janeiro, and $26 \%$ of seroreactive horses were found in the Itaguai microregion ${ }^{14-16}$. The frequency of $N$. risticii reported in southern Brazil through molecular tests such as nested PCR was $1.75 \%{ }^{13}$, a frequency close to that observed in the present study $(2.67 \%$, $\mathrm{n}=5 / 187)$. 
The $N$. risticii-positive horses found in the state of Rio de Janeiro presented some clinicopathological abnormalities that were compatible with PHF signs described in the literature. The pathological process of N. risticii causes lesions in the intestinal musculature, which may explain the biochemical abnormalities in the horses' blood, such as the high levels of $\mathrm{CK}$ and high activity of $\mathrm{LDH}$ in all infected animals. These injuries may also cause leakage of albumin through the inflamed gut wall into the lumen, which may explain the hypoalbuminemia in all infected horses. Hypoalbuminemia and high levels of CK were previously observed in animals with PHF ${ }^{6}$. In that report, the significance of CK activity in the pathology was unclear, but decreased tissue perfusion may lead to acute muscle injury. Fibrinogen increases in the acute phase response and may occur as a result of inflammation of the lesions caused by the pathological process of N. risticii, which also leads to leukocytosis in some cases?. Although the biochemical changes in positive animals are marked, there is no significant difference when compared to the negative population. In this context, is possible that N. risticii infection in horses from the Southeast region is asymptomatic or presents minor clinical or/and laboratory changes.

The phylogenetic analysis showed that the sequences obtained from horses in Rio de Janeiro clustered together with other N. risticii sequences previously reported in bats (Tadarida brasiliensis) from Buenos Aires, Argentina ${ }^{12}$. These data suggested that the genotype of the bacterium does not seem to be influenced by the host. The evolutionary model produced showed that the $N$. risticii clade was composed of three distinct subclades: Genotypes $\mathrm{A}, \mathrm{B}$, and $\mathrm{C}$. The genetic divergence of $\mathrm{N}$. risticii sequences seemed to be related to the geographical location of the samples since a new third genotype from South America, which diverged from the 2 genotypes from North America, was detected in the present study. Previous studies reported such geographical segregation in the United States, showing that sequences from California and Oregon were distinct from sequences from Ohio, Maryland, and Pennsylvania and the sequence from Bueno Aires formed its own cluster ${ }^{12,20,21}$. Thus, genotype seems to be influenced by the unique environmental conditions of each area.

The $16 \mathrm{~S}$ rDNA is widely used for bacterial disease diagnosis ${ }^{18}$. Additionally, the $16 \mathrm{~S}$ rDNA sequence is highly conserved, which allows the creation of a bacterial profile that increases the understanding of the relationship between the genetic divergence and geographic distribution of $N$. risticii. For this reason, the current study chose to characterize the $N$. risticii samples from Brazil with this molecular marker. However, further studies targeting other molecular markers, such as $51 \mathrm{kDa}$ protein (p51), the groESL operon and the citrate synthase gene (gltA), are necessary to improve the genetic characterization of Brazilian samples ${ }^{11}$.

Complete 16S rDNA gene sequences present nine hypervariable regions that are separated by conserved regions $^{22}$. For the hypervariable regions of the $16 \mathrm{~S}$ rDNA sequence to be useful for taxonomic classification and/ or the identification of an organism at the species level, two divergent organisms cannot have identical or highly similar regions. In microbial community studies, the V3 and V6 regions are important in hypervariable regions for the taxonomic identification and distinction of bacterial groups at the genus level ${ }^{23}$. In the present study, the hypervariable region V4 distinguished the different Neorickettsia species, as evidenced by the dendrograms constructed according to the hypervariable region (Supplementary Figs. S2-S6). However, this region was unable to distinguish N. risticii at the genotype level, as evidenced by the phylogenetic reconstruction using the full-length sequences (Fig. 2). In this case, V4 performed well in distinguishing Neorickettsia at the species level. The inability of the V4 region to distinguish $N$. risticii at the genotype level may have been related to the substantial number of point polymorphisms outside of variable regions that, by default, lead to a loss of information ${ }^{24}$. The method of using geodesic distance to analyse the V4 hypervariable region of $16 \mathrm{~S}$ rDNA sequences was the most reliable for representing complete sequences in phylogenetic analysis in previous studies, which was also observed in this study using the $16 \mathrm{~S}$ rDNA sequences of Neorickettsia spp. ${ }^{25}$.

Available data regarding Potomac horse fever are scarce in Brazil, probably because aetiologies for equine enterocolitis has been poorly investigated. Confirmation of the presence of $N$. risticii in horses from the state of Rio de Janeiro is important for raising awareness of the agent, which is in circulation in the Southeast Region of Brazil and highlights the importance of implementing adequate preventive measures to reduce economic losses. Besides, this report shows the need for additional studies to gather further knowledge in order to elucidate the ecology, transmission, and epidemiology of $N$. risticii, as there is no concise information on the biological cycle involved in the transmission of this pathogen in the region.

\section{Material and Methods}

Animals and sampling procedures. A total of 187 blood samples were collected from horses that participated in the herbivorous rabies vaccination campaign carried out by the Angra dos Reis municipal government $\left(-23^{\circ} 00^{\prime} 24.01^{\prime \prime} \mathrm{S} ;-44^{\circ} 19^{\prime} 5.02^{\prime \prime} \mathrm{W}\right)$ in Rio de Janeiro in July and August of 2012 (Fig. 1). After receiving the consent of the animal's owner, one tube containing ethylenediaminetetraacetic acid (EDTA) was used to collect a blood sample from each animal. Then, pathogen screening using molecular methods was performed. A tube without EDTA was used to obtain serum samples.

Haematological analysis. Haematological analysis was performed using a Poch-100iv haematology analyzer (Roche, United States) to obtain horses' red blood cell count (RBC), haemoglobin (Hb), haematocrit (HT), mean corpuscular volume (MCV), mean corpuscular haemoglobin concentration (MCHC), number of platelets (PLT), and white blood cell count (WBC). Blood smears were stained using Diff-Quick stain (Hemacolor, Merck, Brazil) and examined under a light microscope for specific leucometry. The determination of fibrinogen (FIBRI) was performed through manual refractometry using precipitation by heating samples in a water bath at $57^{\circ} \mathrm{C}$ for three minutes ${ }^{26}$.

Biochemical analysis. Samples collected without EDTA were centrifuged at $3000 \mathrm{rpm}$ for $10 \mathrm{~min}$ to obtain the serum. Biochemical measurements were taken using a semi-automatic spectrophotometer (Bioplus $2000^{\circledR}$, Barueri, SP, Brazil) using commercial kits (Labtest, Lagoa Santa, MG, Brazil). The following parameters were 
analysed: aspartate aminotransferase (AST), creatine kinase (CK), lactate dehydrogenase (LDH), total protein (TP), albumin, urea, and creatinine. For internal quality control of biochemical dosages in the Bioplus $2000^{\circledR}$, the control serum Qualitrol $1 \mathrm{H}$ (Labtest) was used.

DNA extraction. DNA was extracted from EDTA blood samples using a GFX Genomic Blood Purification Mini Spin Mini Kit (GE Healthcare Life Science, SP, Brazil) following the manufacturer's recommendations. Quantification was performed through spectrophotometry using a NanoDrop ${ }^{\circledR}$ 2000C instrument (Thermo Scientific, SP, Brazil).

Standard controls. The N. risticii-positive standard control was acquired from commercial slides prepared for immunofluorescence (Fuller Laboratories, Fullerton, CA, USA). Genomic material on the slides was purified using a DNeasy Blood and Tissue Kit (Qiagen, Valencia, CA, USA) according to the manufacturer's recommendations.

Nuclease-free water (Ambion ${ }^{\circledR}$, Thermo Scientific, Wilmington, DE, USA) and a negative equine blood sample were used as a negative amplification control.

Molecular detection. To perform initial pathogen screening, DNA samples extracted from equine whole blood were submitted to $N$. risticii-specific real-time quantitative PCR (qPCR) with a detection limit of 10 copies of $N$. risticii $16 \mathrm{~S} \mathrm{rDNA}^{27}$. The oligonucleotide probes used were the forward probe ER.133f (5'-GTTATTCCCTACTACCAGGCAAGTTC-3'), the reverse probe ER.54r (5'-AACGGAATCAGGGCTGCTT-3'), and the quencher-labelled probe ER.77p (FAM-ACGCACCCGTCTGCCACGGGA-TAMRA). All samples were tested using a TaqMan ${ }^{\circledR}$ Exogenous Internal Positive Control Reagents Kit (Applied Biosystems, Foster City, CA) following the manufacturer's instructions. Reactions were performed using the following reagents in a total volume of $12 \mu \mathrm{l}$ : 1X TaqMan Universal PCR Master Mix (Applied Biosystems), $400 \mathrm{nM}$ each primer, $800 \mathrm{nM}$ probe, and $1 \mu \mathrm{l}$ of target DNA. The amplification conditions were as follows: $5 \mathrm{~min}$ at $95^{\circ} \mathrm{C}$ and 45 cycles of $15 \mathrm{~s}$ at $95^{\circ} \mathrm{C}$ and $60 \mathrm{~s}$ at $60^{\circ} \mathrm{C}$ in a Step One instrument (Applied Biosystems). Samples with cycle quantification ("Cq") less than or equal to 40 were considered positive.

The five positive samples from qPCR were submitted to conventional PCR to amplify the complete $16 \mathrm{~S}$ rDNA region $(1470 \mathrm{bp})^{28}$. The oligonucleotide probes used were the forward probe N16-25F (5'-TCAGAACGAACGCTAGCGGT-3') and the reverse probe N1500R ( $5^{\prime}$-AAAGGAGGTAATCCAGCCGCAGGTTCAC- $\left.3^{\prime}\right)$. The amplification conditions were as follows: 5 min at $95^{\circ} \mathrm{C}$, followed by 40 cycles of $15 \mathrm{~s}$ at $95^{\circ} \mathrm{C}, 60 \mathrm{~s}$ at $58^{\circ} \mathrm{C}$, and $45 \mathrm{~s}$ at $72^{\circ} \mathrm{C}$, on a Veriti instrument (Applied Biosystems).

Sequencing. Amplicons were purified with a Clean Sweep enzymatic purification kit (Applied Biosystem) according to the manufacturer's recommendations. Sequencing was conducted using the Sanger method with an ABI 3730 DNA analyzer (Applied Biosystems).

Phylogenetic analysis. The five sequences obtained were analysed with DNA Sequence Assembler v4 (Heracle BioSoft, Arges, Romania) and submitted to the Basic Local Alignment Search Tool (BLAST) algorithm to verify the similarity of the samples to nucleotide sequences available in GenBank. An initial dataset was assembled with the partial sequences of $16 \mathrm{~S}$ rDNA obtained in this study and a set of nearly complete sequences of Neorickettsia spp. (1455-1342 bp). Sequences were aligned using the ClustalW algorithm in MEGA7 software (Molecular Evolutionary Genetics Analysis Version 7.0 for Bigger Datasets) ${ }^{29}$. The complete 16S rDNA sequence of $N$. risticii accession number NR074389 was used as a reference for alignment.

Phylogenetic analysis was performed using the maximum likelihood method based on the Kimura 2-parameter model ${ }^{30}$. The tree with the largest log-likelihood $(-2088.28)$ is shown in Fig. 2 . There were a total of 1282 positions in the final dataset. A bootstrap test with 1000 pseudo-replicates was used for the confidence analysis of the clades. The $16 \mathrm{~S}$ rDNA sequence of Neorickettsia helminthoeca (KX462531) was used as an outgroup for the phylogenetic analysis. In this study, monophyletic clades with $0.3 \%$ evolutionary divergence between clades and large bootstrap values were considered distinct genotypes of $N$. risticii. Evolutionary analyses were conducted in MEGA X.

A second dataset was assembled with only $16 \mathrm{~S}$ rDNA sequences from Neorickettsia spp. of over 1200 bp available in GenBank and the SILVA database. Phylogenetic reconstruction was performed using the same methods described above. In this dataset, variable regions of $16 \mathrm{~S}$ rDNA fragments of Neorickettsia were studied. The 16S rDNA regions were determined according to a previously published protocol, followed by a visual analysis of the sequence alignment of Neorickettsia species ${ }^{25}$. Hypervariable regions were identified through entropy analysis using BioEdit version 7.0.9. $0^{31}$. After alignment, a dendrogram was built using the maximum likelihood method based on the Kimura 2-parameter model for each variable region. The hypervariable region of the 16S rDNA sequence of Neorickettsia spp. that best represented the phylogenetic reconstruction obtained with the complete $16 \mathrm{~S}$ rDNA sequence was also determined.

Ethical statement. All experimental protocols used in this study were approved by the Committee on Ethics in the Use of Animals (CEUA) of the Federal University of Fluminense under number 66/11. In addition, all methods applied in this investigation were in accordance with relevant guidelines and regulations of the Rural Federal University of Rio de Janeiro. 


\section{Data availability}

Sequences were deposited in GenBank with accession numbers from MK760556 to MK760560. https://www. ncbi.nlm.nih.gov/nuccore/MK760556.1/; https://www.ncbi.nlm.nih.gov/nuccore/MK760557.1/; https://www. ncbi.nlm.nih.gov/nuccore/MK760558.1/; https://www.ncbi.nlm.nih.gov/nuccore/MK760559.1/; https://www. ncbi.nlm.nih.gov/nuccore/MK760560.1/.

Received: 30 December 2019; Accepted: 10 April 2020;

Published online: 29 April 2020

\section{References}

1. Holland, C. J. et al. Isolation, experimental transmission, and characterization of the causative agent of Potomac horse fever. Science 35, 524-526, https://doi.org/10.1126/science.3880925 (1985).

2. Marutani, V. H. B., Headley, S. A. \& Bracarense, A. P. F. R. L. Putative Equine Neorickettsiosis in a Mare from Southern Brazil. Acta Scientiae Veterinariae 47, 451, https://doi.org/10.22456/1679-9216.97586 (2019).

3. Xiong, Q. et al. An Ecotype of Neorickettsia risticii Causing Potomac Horse Fever in Canada. Appl. Env. Microbiol. 82, 6030-6036, https://doi.org/10.1128/AEM.01366-16 (2016).

4. Lin, M., Zhang, C., Gibson, K. \& Rikihisa, Y. Analysis of complete genome sequence of Neorickettsia risticii: causative agent of Potomac horse fever. Nucleic Acids Res. 37, 6076-6091, https://doi.org/10.1093/nar/gkp642 (2009).

5. Shaw, S. D. \& Stämpfli, H. Diagnosis and Treatment of Undifferentiated and Infectious Acute Diarrhea in the Adult Horse. Vet. Clin. North. Am. Equine Pract. 34, 39-53, https://doi.org/10.1016/j.cveq.2017.11.002 (2018).

6. Bertin, F. R. et al. Clinical and Clinicopathological Factors Associated with Survival in 44 Horses with Equine Neorickettsiosis (Potomac Horse Fever). J. Vet. Int. Med. 27, 1528-1534, https://doi.org/10.1111/jvim.12209 (2013).

7. Coimbra, H. S. \& Schuch, L. F. D. Veitenheimer MIL, Meireles MCA. Neorickettsia (Ehrlichia) risticii no Sul do Brasil: Heleobia spp. (Mollusca: Hydrobilidae) e Parapleurolophocecous cercariae (Trematoda: Digenea) como possíveis vetores. Arq. Inst. Biol. 72, 325-329 (2005).

8. Dutra, F. et al. Equine monocytic Ehrlichiosis (Potomac horse fever) in 51 horses in Uruguay and southern Brazil. J. Vet. Diagn. Invest. 13, 433-437, https://doi.org/10.1177/104063870101300514 (2001).

9. Heller, M. C. et al. Two cases of Neorickettsia (Ehrlichia) risticii infection in horses from Nova Scotia. Can Vet J. 45, 421-3 (2004).

10. Cicuttin, G. L. et al. Molecular detection of Neorickettsia risticii in Brazilian free-tailed bats (Tadarida brasiliensis) from Buenos Aires, Argentina. Pesqui. Vet. Bras. 33, 648-650, https://doi.org/10.1590/S0100-736X2013000500016 (2013).

11. Cicuttin, G. L. et al. Neorickettsia risticii, Rickettsia sp. and Bartonella sp. in Tadarida brasiliensis bats from Buenos Aires, Argentina, Comp. Immunol. Microbiol. Infect. Dis. 52, 1-5, https://doi.org/10.1016/j.cimid.2017.04.004. (2017).

12. Cicuttin, G. L. \& Greiman, S. E. Análisis filogenético de Neorickettsia risticii detectada em murciélagos de Argentina. InVet. 18, 341-347 (2016).

13. Coimbra, H. S. et al. Ehrliquiose Monocítica Equina no Rio Grande do Sul: aspectos clínicos, anatomopatológicos e epidemiológicos. Pesqui. Vet. Bras. 26, 97-101, https://doi.org/10.1590/S0100-736X2006000200006 (2006).

14. Ferrão, C. M. et al. Equine monocytic ehrlichiosis (EME) in Rio de Janeiro State, Brazil. Arq. Bras. Med. Vet. Zootec. 59, 1575-1578, https://doi.org/10.1590/S0102-09352007000600034 (2007).

15. Moreira, L. M. C. et al. Frequency of equine monocytic ehrlichiosis (EME) in Brazil. Pesqui. Vet. Bras. 33, 697-699, https://doi. org/10.1590/S0100-736X2013000600001 (2013).

16. Roier, E. C. R. et al. Epidemiological survey of Neorickettsia risticii in equids from the State of Rio de Janeiro, Brazil. Pesqui. Vet. Bras. 36, 939-946, https://doi.org/10.1590/s0100-736X2016001000004 (2016).

17. Dumler, J. S. et al. Reorganization of Genera in the Families Rickettsiaceae and Anaplasmataceae in the Order Rickettsiales: Unification of Some Species of Ehrlichia with Anaplasma, Cowdria With Ehrlichia and Ehrlichia With Neorickettsia, Descriptions of Six New Species Combinations and Designation of Ehrlichia Equi and 'HGE Agent' as Subjective Synonyms of Ehrlichia phagocytophila. Int. J. Syst. Evol. Microbiol. 51, 2145-2165 (2001).

18. Stackebrandt, E. \& Goebel, B. M. Taxonomic Note: A Place for DNA-DNA Reassociation and 16S rRNA Sequence Analysis in the Present Species Definition in Bacteriology. Int. J. Syst. Evol. Microbiol. 44(4), 846-849, https://doi.org/10.1099/00207713-44-4-846 (1994).

19. Marcolongo-Pereira, C. et al. Doenças de equinos na região Sul do Rio Grande do Sul. Pesqui. Vet. Bras. 34, 205-210, https://doi. org/10.1590/S0100-736X2014000300002 (2014).

20. Kanter, M. et al. Analysis of 16S 320 rRNA and 51 Kilodalton Antigen Gene and Transmission in Mice of Ehrlichia risticii in Virgulate Trematodes from Elimia livescens Snails in Ohio. J. Clin. Microbiol. 38, 3349-3358 (2000).

21. Mott, J. et al. Molecular analysis of Neorickettsia risticii in adult aquatic insects in Pennsylvania, in horses infected by ingestion of insects, and isolated in cell culture. J. Clin. Microbiol. 40, 690-693, https://doi.org/10.1128/jcm.40.2.690-693.2002 (2002).

22. Chakravorty, S. et al. A detailed analysis of $16 \mathrm{~S}$ ribosomal RNA gene segments for the diagnosis of pathogenic bacteria. J. Microbiol. Methods. 69(2), 330-339, https://doi.org/10.1016/j.mimet.2007.02.005 (2007).

23. Huse, L. D. et al. Exploring Microbial Diversity and Taxonomy Using SSU rRNA Hypervariable Tag Sequencing. PLoS Genet. 4(11), e1000255 (2008).

24. Stackebrandt, E., Liesack, W., Witt, D. \& Ribosomal, R. N. A. and rRNA sequence analysis. Gene. 115, 255-260, https://doi. org/10.1016/0378-1119(92)90567-9 (1992).

25. Yang, B., Wang, Y. \& Qian, P. Y. Sensitivity and correlation of hypervariable regions in $16 \mathrm{~S}$ rRNA genes in phylogenetic analysis. BMC Bioinformatics. 17, 135, https://doi.org/10.1186/s12859-016-0992-y (2016).

26. Taylor S. D. Neorickettsia risticii. In: Pusterla N, Higgins, J. Interpretation of equine laboratory diagnostics John Wiley \& Sons, Inc. pp. 177-180. (2018).

27. Pusterla, N. et al. Detection and quantitation of Ehrlichia risticii genomic DNA in infected horses and snails by real-time PCR. Vet. Parasit. 90, 129-135, https://doi.org/10.1016/S0304-4017(00)00227-2 (2009).

28. Greiman, S. E. Large scale screening of digeneans for Neorickettsia endosymbionts using real-time PCR reveals new Neoricekttsia genotypes, host associations and geographic records including first reports from Australia and China. PLoS One. 9, e98452, https:// doi.org/10.1371/journal.pone.0098453 (2014).

29. Kumar, S., Stecher, G., Li, M., Knyaz, C. \& Tamura, K. MEGA X: Molecular Evolutionary Genetics Analysis across computing platforms. Mol. Bio Evol. 35, 1547-1549, https://doi.org/10.1093/molbev/msw054 (2018).

30. Kimura, M. A simple method for estimating the evolutionary 348 rate of base substitutions through comparative studies of nucleotide sequences. J. Mol. Evol. 16, 111-120, https://doi.org/10.1007/BF01731581 (1980).

31. Hall, T. A. BioEdit: a user-friendly biological sequence alignment editor and analysis program for Windows 95/98/NT. Nucleic Acids Symp. Ser. 41, 95-98 (1999). 


\section{Acknowledgements}

The authors disclose receipt of the following financial support for the research, authorship, and/or publication of this article. The National Council for Scientific and Technological Development and the 'Carlos Chagas Filho' Foundation for Research Support of the State of Rio de Janeiro.

\section{Author contributions}

C.M., N.A., AM originally formulated the idea, R.O., A.M. and V.V. collected samples, R.O. and A.M. performed the Complete blood count $(\mathrm{CBC})$ and biochemistry measurements, C.S. and M.P. conducted extractions and sequencing, C.B. and A.G. analysed the data and P.G.P. and H.S. wrote the manuscript.

\section{Competing interests}

The authors declare no competing interests.

\section{Additional information}

Supplementary information is available for this paper at https://doi.org/10.1038/s41598-020-64328-2.

Correspondence and requests for materials should be addressed to P.G.P.

Reprints and permissions information is available at www.nature.com/reprints.

Publisher's note Springer Nature remains neutral with regard to jurisdictional claims in published maps and institutional affiliations.

(c) (i) Open Access This article is licensed under a Creative Commons Attribution 4.0 International License, which permits use, sharing, adaptation, distribution and reproduction in any medium or format, as long as you give appropriate credit to the original author(s) and the source, provide a link to the Creative Commons license, and indicate if changes were made. The images or other third party material in this article are included in the article's Creative Commons license, unless indicated otherwise in a credit line to the material. If material is not included in the article's Creative Commons license and your intended use is not permitted by statutory regulation or exceeds the permitted use, you will need to obtain permission directly from the copyright holder. To view a copy of this license, visit http://creativecommons.org/licenses/by/4.0/.

(c) The Author(s) 2020 